\title{
SUPPLEMENTARY MATERIAL: CLUSTERING CORRELATED, SPARSE DATA STREAMS TO ESTIMATE A LOCALIZED HOUSING PRICE INDEX
}

\author{
By You Ren*, Emily B. Fox*, And Andrew Bruce* \\ University of Washington*
}

\section{APPENDIX A: CODE AND DATA}

The code for running our algorithm on a simulated dataset is available on Github: https://github.com/shirleyuw/hyperlocalHouseIndex.

The data used in this study was provided by the Zillow Group. Under the terms of the contract, we are not able to share this data publicly. However, similar housing data can be obtained directly from King County at http://info.kingcounty.gov/assessor/DataDownload/default.aspx and http://www5.kingcounty.gov/gisdataportal/. The first website gives housing sales data and property characteristics of all residences in King County. The parcel latitude and longitude for each residence can be extracted from the second website. As long as the usage of the data is non-commercial, it is freely available and can be downloaded from the King County websites.

\section{APPENDIX B: CONDITIONAL LIKELIHOOD OF DATA IN CLUSTER}

In this section, we describe how to compute the likelihood of the data from all time series assigned to a given cluster $k$, conditioned on the model parameters. We consider two mathematically equivalent methods: one based on the collection of observations directly, and the other using sufficient statistics of the observed house sales. In what follows, we drop the cluster index $k$ for simplicity of notation.

B.1. Naive Kalman filtering. We consider a straightforward extension of the standard Kalman filter recursions to compute the marginal likelihood of all observations in cluster $k$ when there can be multiple observations per time step. The derivation is as follows. The cluster marginal likelihood can be calculated as

$$
\log P\left(\mathbf{y}_{1: T}\right)=\sum_{t=1}^{T} \log P\left(\mathbf{y}_{t} \mid \mathbf{y}_{1: t-1}\right),
$$

where the distribution of new observations at time $t$ conditional on past time series is

$$
\begin{gathered}
\mathbf{y}_{t} \mid \mathbf{y}_{1: t-1} \sim \mathcal{N}\left(\mathbf{y}_{t} \mid C_{t} \mu_{t \mid t-1}+D_{t} U_{t}, S_{t}\right) \\
1 \\
\text { imsart-aoas ver. 2014/02/20 file: Appendix.tex date: March 27, } 2017
\end{gathered}
$$


The quantities $\mu_{t \mid t-1}$ and $S_{t}$ are obtained by the Kalman filter:

$$
\begin{aligned}
\text { Predict } \mu_{t \mid t-1} & =A \mu_{t-1 \mid t-1} \\
V_{t \mid t-1} & =A V_{t-1 \mid t-1} A^{T}+Q \\
\text { Calculate } \quad S_{t} & =C_{t} V_{t \mid t-1} C_{t}^{T}+R_{t} \\
\text { Kalman gain matrix } \quad K_{t} & =V_{t \mid t-1} C_{t}^{T} S_{t}^{-1} \\
\text { Filter } \quad \mu_{t \mid t} & =\mu_{t \mid t-1}+K_{t}\left(\mathbf{y}_{t}-C_{t} \mu_{t \mid t-1}-D_{t} U_{t}\right) \\
V_{t \mid t} & =\left(\mathbf{I}-K_{t} C_{t}\right) V_{t \mid t-1}
\end{aligned}
$$

The coefficient matrix $C_{t}$ is an indicator matrix mapping each observation to its specific census tract. The matrix $D_{t}$ is a coefficient matrix for hedonic effects. The filter should be applied to data for all tracts in cluster $k$ together.

The purpose of doing filtering here is to evaluate the conditional likelihood of tract $i$ belonging to cluster $k$, given observations of all the other tracts in cluster $k$. The conditional likelihood is

$$
\begin{aligned}
\text { (B.4) } P\left(\mathbf{y}_{1: T, i} \mid \mathbf{y}_{1: T,-i}\right)= & P\left(\mathbf{y}_{1, i} \mid \mathbf{y}_{1,-i}\right) P\left(\mathbf{y}_{2, i} \mid \mathbf{y}_{1}, \mathbf{y}_{2,-i}\right) \\
& P\left(\mathbf{y}_{3, i} \mid \mathbf{y}_{1: 2}, \mathbf{y}_{3,-i}\right) \cdots P\left(\mathbf{y}_{T, i} \mid \mathbf{y}_{1: T-1}, \mathbf{y}_{T,-i}\right) .
\end{aligned}
$$

Therefore the log-likelihood of observations for tract $i$ conditional on the other observations in cluster $k$ is

$$
\log P\left(\mathbf{y}_{1: T, i} \mid \mathbf{y}_{1: T,-i}\right)=\sum_{t=1}^{T} \log P\left(\mathbf{y}_{t, i} \mid \mathbf{y}_{1: t-1}, \mathbf{y}_{t,-i}\right) .
$$

At time $t$, we have the joint distribution $\mathbf{y}_{t, i}, \mathbf{y}_{t,-i} \mid \mathbf{y}_{1: t-1}$, which is $\mathbf{y}_{t} \mid \mathbf{y}_{1: t-1}$ in Eq. (B.2). We can then derive the conditional distribution $\mathbf{y}_{t, i} \mid \mathbf{y}_{1: t-1}, \mathbf{y}_{t,-i}$ by the conventional conditional multivariate normal distribution as follows:

$$
A \mid B \sim \mathcal{N}\left(\mu_{A}+\Sigma_{A B} \Sigma_{B B}^{-1}\left(B-\mu_{B}\right), \Sigma_{A A}-\Sigma_{A B} \Sigma_{B B}^{-1} \Sigma_{B A}\right)
$$

for the general form of a joint multivariate normal distribution

$$
\left(\begin{array}{c}
A \\
B
\end{array}\right) \sim \mathcal{N}\left[\left(\begin{array}{c}
\mu_{A} \\
\mu_{B}
\end{array}\right),\left(\begin{array}{c}
\Sigma_{A A}, \Sigma_{A B} \\
\Sigma_{B A}, \Sigma_{B B}
\end{array}\right)\right]
$$

B.2. Sufficient statistic Kalman filter. If all $p^{(k)}$ tracts in a particular cluster $k$ have observations at time $t$, the sufficient statistic multivariate 
Kalman filter algorithm is as follows:

$$
\begin{aligned}
\text { Predict } \mu_{t \mid t-1} & =A \mu_{t-1 \mid t-1} \\
V_{t \mid t-1} & =A V_{t-1 \mid t-1} A^{T}+Q \\
\text { Calculate } \quad S_{t} & =V_{t \mid t-1}+\bar{R}_{t} \\
\text { (Kalman gain matrix }) \quad K_{t} & =V_{t \mid t-1} S_{t}^{-1} \\
\text { Filter } \quad \mu_{t \mid t} & =\mu_{t \mid t-1}+K_{t}\left(\overline{\dot{\mathbf{y}}}_{\mathbf{t}}-\mu_{\mathbf{t} \mid \mathbf{t}-\mathbf{1}}\right) \\
V_{t \mid t} & =\left(\mathbf{I}-K_{t}\right) V_{t \mid t-1}
\end{aligned}
$$

where $\dot{\mathbf{y}}_{t}$ denotes the vector of observations with hedonic effects removed and $\overline{\dot{y}}_{\mathbf{t}}$ the tract-specific mean of all observations at time $t$ after removing hedonic effects. The matrix $\bar{R}_{t}$ is the diagonal matrix of size $p^{(k)}$-by- $p^{(k)}$ with $(i, i)$-th entry being $\sigma_{i}^{2} / L_{t, i}$. The variable $\sigma_{i}^{2}$ is the observational variance for tract $i$ and the variable $L_{t, i}$ is the number of observations in tract $i$ at time $t$. Note that all matrix operations above are of the size of the cluster, $p^{(k)}$. If some tracts at time $t$ have no transactions, i.e. $L_{t, i}=0$, we use the following recursion instead:

$$
\begin{aligned}
\text { Predict } \mu_{t \mid t-1} & =A \mu_{t-1 \mid t-1} \\
V_{t \mid t-1} & =A V_{t-1 \mid t-1} A^{T}+Q \\
\text { Calculate } \quad S_{t} & =\bar{C}_{t} V_{t \mid t-1} \bar{C}_{t}^{T}+\bar{R}_{t} \\
\text { (Kalman gain matrix) } K_{t} & =V_{t \mid t-1} \bar{C}_{t}^{T} S_{t}^{-1} \\
\text { Filter } \quad \mu_{t \mid t} & =\mu_{t \mid t-1}+K_{t}\left(\overline{\mathbf{y}}_{\mathbf{t}}-\mu_{\mathbf{t} \mid \mathbf{t}-\mathbf{1}}\right) \\
V_{t \mid t} & =\left(\mathbf{I}-K_{t} \bar{C}_{t}\right) V_{t \mid t-1}
\end{aligned}
$$

In the formula above, $\bar{C}_{t}$ is an indicator matrix of non-zero sales at time $t$. The matrix has size $p^{(k)}, \times p^{(k)}$, where $p^{(k)}$, is the number of tracts that have observations at time $t$ (therefore $\left.p^{(k)}, \leq p^{(k)}\right)$. The response variance matrix $\bar{R}_{t}$ is of size $p^{(k)}, \times p^{(k)}$, and includes the variance for tracts that have observations.

\section{APPENDIX C: DERIVATION OF SAMPLING STEPS}

In this section, we provide further details and derivations of the sampling steps outlined in Section 4 of the main paper.

C.1. Forward filter backward sampler for the intrinsic price dynamics. To sample the latent state sequence, we run a forward filter backward sampler. 


\section{Forward Kalman Filter}

1. Initialize filter with $\mu_{0 \mid 0}, V_{0 \mid 0}$, where $X_{0} \sim N\left(\mu_{0 \mid 0}, V_{0 \mid 0}\right)$

2. Working forward in time, for $t=1, \cdots, T$, implement the sufficient statistic filter of Appendix B.2 to obtain $\mu_{t \mid t}, V_{t \mid t}$ for $t=1, \cdots, T$, where $X_{t} \mid \mathbf{y}_{1: t} \sim N\left(\mu_{t \mid t}, V_{t \mid t}\right)$.

\section{Backward Sampler}

1. Draw $X_{T}$ from $P\left(X_{T} \mid \mathbf{y}_{1: T}\right)=N\left(\mu_{T \mid T}, V_{T \mid T}\right)$.

2. Sequentially sample backward, for $t=T-1, \cdots, 0, x_{t}$ from $P\left(X_{t} \mid x_{t+1}, \mathbf{y}_{1: t}\right)$ :

(C.1) $x_{t} \sim N\left[\mu_{t \mid t}+J_{t}\left(x_{t+1}-\mu_{t+1 \mid t}\right), \quad V_{t \mid t}-J_{t} V_{t+1 \mid t} J_{t}^{T}\right]$

where $J_{t}=V_{t \mid t} A^{T} V_{t+1 \mid t}^{-1}$.

C.2. Sampling the latent factor $\boldsymbol{\eta}^{*}$. For any $t$, the vector of latent states for all $p$ tracts jointly follows a vector autoregressive (VAR) process as follows:

$$
\left[\begin{array}{l}
x_{t, 1} \\
\vdots \\
x_{t, n}
\end{array}\right]=\left(\begin{array}{lll}
a_{1} & & 0 \\
& \ddots & \\
0 & & a_{n}
\end{array}\right)\left[\begin{array}{l}
x_{t-1,1} \\
\vdots \\
x_{t-1, n}
\end{array}\right]+(\Lambda \cdot Z)\left[\begin{array}{l}
\eta_{t, 1}^{*} \\
\vdots \\
\eta_{t, K}^{*}
\end{array}\right]+\tilde{\boldsymbol{\epsilon}}_{t}
$$

The VAR process can be written in the form of vectors and matrices:

$$
\mathbf{x}_{\mathbf{t}}=A \mathbf{x}_{\mathbf{t}-\mathbf{1}}+(\Lambda \cdot Z) \boldsymbol{\eta}_{t}^{*}+\tilde{\boldsymbol{\epsilon}}_{t}
$$

such that

$$
\mathbf{x}_{\mathbf{t}}-A \mathbf{x}_{\mathbf{t}-\mathbf{1}} \sim \mathcal{N}_{n}\left[(\Lambda \cdot Z) \boldsymbol{\eta}_{t}^{*}, \sigma_{0}^{2} I_{n}\right] .
$$

By first multiplying $(\Lambda \cdot Z)^{T}$, we get

$$
(\Lambda \cdot Z)^{T}\left(\mathbf{x}_{\mathbf{t}}-A \mathbf{x}_{\mathbf{t}-\mathbf{1}}\right) \sim \mathcal{N}_{K}\left[(\Lambda \cdot Z)^{T}(\Lambda \cdot Z) \boldsymbol{\eta}_{t}^{*}, \sigma_{0}^{2}(\Lambda \cdot Z)^{T}(\Lambda \cdot Z)\right] .
$$

We then multiply by $\left[(\Lambda \cdot Z)^{T}(\Lambda \cdot Z)\right]^{-1}$ and obtain

$$
\left[(\Lambda \cdot Z)^{T}(\Lambda \cdot Z)\right]^{-1}(\Lambda \cdot Z)^{T}\left(\mathbf{x}_{\mathbf{t}}-A \mathbf{x}_{\mathbf{t}-1}\right) \sim \mathcal{N}_{K}\left\{\boldsymbol{\eta}_{t}^{*}, \sigma_{0}^{2}\left[(\Lambda \cdot Z)^{T}(\Lambda \cdot Z)\right]^{-1}\right\} .
$$

Given the prior of $\boldsymbol{\eta}_{t}^{*} \sim \mathcal{N}_{K}\left(\mathbf{0}, I_{K}\right)$ and the likelihood in Eq. (C.6), by conjugacy, the full conditional distribution for $\boldsymbol{\eta}_{t}^{*}$ is

$$
\boldsymbol{\eta}_{t}^{*} \mid \boldsymbol{\lambda}, \mathbf{z}, \mathbf{x}, \sigma_{0}^{2} \sim \mathcal{N}_{K}\left\{\begin{array}{c}
\Omega \frac{1}{\sigma_{0}^{2}}(\Lambda \cdot Z)^{T}\left(\mathbf{x}_{\mathbf{t}}-A \mathbf{x}_{\mathbf{t}-\mathbf{1}}\right), \\
\Omega=\left[I_{K}+\frac{1}{\sigma_{0}^{2}}(\Lambda \cdot Z)^{T}(\Lambda \cdot Z)\right]^{-1}
\end{array}\right\} .
$$


C.3. Sampling the factor loadings $\lambda$. For any $t$,

$$
x_{t, i}=a_{i} x_{t-1, i}+\lambda_{i k} Z_{i k} \eta_{t, k}^{*}+\tilde{\epsilon}_{t, i} .
$$

Recall the definition of the membership matrix $Z$ from Section 3.2. If $Z_{i k}=$ 0 , then the full conditional distribution for $\lambda_{i k}$ is just its prior,

$$
\lambda_{i k} \mid \mathbf{x}, \mathbf{a}, \boldsymbol{\eta}^{*}, \mathbf{z}, \sigma_{0}^{2}, \mu_{\lambda}, \sigma_{\lambda}^{2} \sim \mathcal{N}\left(\mu_{\lambda}, \sigma_{\lambda}^{2}\right)
$$

If $Z_{i k}=1$ then

$$
\begin{aligned}
& p\left(\lambda_{i k} \mid \mathbf{x}, \mathbf{a}, \boldsymbol{\eta}^{*}, \mathbf{z}, \sigma_{0}^{2}, \mu_{\lambda}, \sigma_{\lambda}^{2}\right) \\
\propto & \mathcal{N}\left(\mu_{\lambda}, \sigma_{\lambda}^{2}\right) \prod_{t=1}^{T} \mathcal{N}\left(x_{t, i} \mid a_{i} x_{t-1, i}+\lambda_{i k} \eta_{t, k}^{*}, \sigma_{0}^{2}\right) \\
\propto & \mathcal{N}\left(\mu_{\lambda}, \sigma_{\lambda}^{2}\right) \prod_{t=1}^{T} \mathcal{N}\left(\frac{x_{t, i}-a_{i} x_{t-1, i}}{\eta_{t, k}^{*}} \mid \lambda_{i k}, \frac{\sigma_{0}^{2}}{\eta_{t, k}^{* 2}}\right) \\
\propto & \mathcal{N}\left[\omega\left(\frac{\mu_{\lambda}}{\sigma_{\lambda}^{2}}+\sum_{t=1}^{T} \frac{\left(x_{t, i}-a_{i} x_{t-1, i}\right) / \eta_{t, k}^{*}}{\sigma_{0}^{2} / \eta_{t, k}^{* 2}}\right), \omega=\left(\frac{1}{\sigma_{\lambda}^{2}}+\frac{1}{\sigma_{0}^{2}} \sum_{t=1}^{T} \eta_{t, k}^{* 2}\right)^{-1}\right] .
\end{aligned}
$$

In summary, the full conditional distribution for $\lambda_{i k}$ is

$$
\lambda_{i k} \mid \mathbf{x}, \mathbf{a}, \boldsymbol{\eta}^{*}, \mathbf{z}, \sigma_{0}^{2}, \mu_{\lambda}, \sigma_{\lambda}^{2} \sim \mathcal{N}\left(\mu_{i k}^{*}, v_{i k}^{*}\right)
$$

where

$\left(\mu_{i k}^{*}, v_{i k}^{*}\right)=\left\{\begin{array}{ll}\left(\mu_{\lambda}, \sigma_{\lambda}^{2}\right) & \text { if } Z_{i k}=0 \\ \omega\left(\mu_{\lambda} \frac{1}{\sigma_{\lambda}^{2}}+\frac{1}{\sigma_{0}^{2}} \sum_{t=1}^{T} \epsilon_{t, i} \eta_{t, k}^{*}\right), \omega=\left(\frac{1}{\sigma_{\lambda}^{2}}+\frac{1}{\sigma_{0}^{2}} \sum_{t=1}^{T} \eta_{t, k}^{* 2}\right)^{-1} & \text { if } Z_{i k}=1\end{array}\right.$.

Note that $\epsilon_{t, i}=x_{t, i}-a_{i} x_{t-1, i}$.

C.4. Sampling the autoregressive process parameters $\boldsymbol{a}_{\boldsymbol{i}}, \boldsymbol{\sigma}_{\mathbf{0}}^{\mathbf{2}}$. Combining Eq. (3.3) and Eq. (3.5) of the main paper, for $z_{i}=k$

$$
x_{t, i}=a_{i} x_{t-1, i}+\lambda_{i k} \eta_{t, k}^{*}+\tilde{\epsilon}_{t, i}, \quad \tilde{\epsilon}_{t, i} \sim \mathcal{N}\left(0, \sigma_{0}^{2}\right) .
$$

Therefore,

$$
x_{t, i} \sim \mathcal{N}\left(a_{i} x_{t-1, i}+\lambda_{i k} \eta_{t, k}^{*}, \sigma_{0}^{2}\right) .
$$

By rearranging the terms, we get

(C.13) $\frac{x_{t, i}-\lambda_{i k} \eta_{t, k}^{*}}{x_{t-1, i}} \sim \mathcal{N}\left(a_{i}, \frac{\sigma_{0}^{2}}{x_{t-1, i}^{2}}\right), \quad$ i.i.d. for $t=1, \cdots, T$.

imsart-aoas ver. 2014/02/20 file: Appendix.tex date: March 27, 2017 
By conjugacy, and conditioning on a cluster assignment $z_{i}=k$, the full conditional distribution of the AR process coefficient $a_{i}$ is

$$
\begin{aligned}
p\left(a_{i} \mid z_{i}\right. & \left.=k, \mathbf{x}_{i}, \boldsymbol{\eta}^{*(k)}, \lambda_{i k}, \sigma_{0}^{2}, \mu_{a}, \sigma_{a}^{2}\right) \propto \mathcal{N}\left(a_{i} \mid \mu_{a}, \sigma_{a}^{2}\right) \prod_{t=1}^{T} \mathcal{N}\left[\frac{x_{t, i}-\lambda_{i k} \eta_{t, k}^{*}}{x_{t-1, i}} \mid a_{i}, \frac{\sigma_{0}^{2}}{x_{t-1, i}^{2}}\right] \\
& \propto \mathcal{N}\left(V\left[\frac{1}{\sigma_{a}^{2}} \cdot \mu_{a}+\sum_{t=1}^{T}\left(\frac{x_{t-1, i}^{2}}{\sigma_{0}^{2}} \cdot \frac{x_{t, i}-\lambda_{i k} \eta_{t, k}^{*}}{x_{t-1, i}}\right)\right], V=\left(\frac{1}{\sigma_{a}^{2}}+\sum_{t=1}^{T} \frac{x_{t-1, i}^{2}}{\sigma_{0}^{2}}\right)^{-1}\right) .
\end{aligned}
$$

Again by conjugacy, the variance parameter $\sigma_{0}^{2}$ has full conditional

$$
\begin{aligned}
& \sigma_{0}^{2} \mid \boldsymbol{\lambda}, \boldsymbol{\eta}^{*}, \mathbf{a}, \mathbf{z}, \mathbf{x}, \alpha_{\epsilon 0}, \beta_{\epsilon 0} \\
\sim & \operatorname{IG}\left(\alpha_{\epsilon 0}+\frac{T p}{2}, \beta_{\epsilon 0}+\frac{1}{2} \sum_{t=1}^{T} \sum_{i=1}^{p} \sum_{k=1}^{K} z_{i k}\left(x_{t, i}-a_{i} x_{t-1, i}-\lambda_{i k} \eta_{t, k}^{*}\right)^{2}\right) .
\end{aligned}
$$

C.5. Sampling emission parameters $\boldsymbol{R}, \boldsymbol{\beta}$. By conjugacy, we can sample the observation variance $R_{i}$ for $i=1, \cdots, p$ as

$R_{i} \mid \mathbf{x}_{1: T, i}, \mathbf{y}_{1: T, i}, \alpha_{R 0}, \beta_{R 0} \sim \mathrm{IG}\left(\alpha_{R 0}+\frac{1}{2} m_{i}, \beta_{R 0}+\frac{1}{2} \sum_{t=1}^{T} \sum_{l=1}^{L t}\left(y_{t, i, l}-x_{t, i}\right)^{2}\right)$,

where $m_{i}$ is the number of transactions in census tract $i$.

For tract $i$ and hedonic covariate $h$, the posterior distribution for covariate effect $\beta_{i, h}$ is

(C.17) $p\left(\beta_{i, h} \mid R_{i}, \mathbf{x}_{1: T, i}, \mathbf{y}_{1: T, i}, \mu_{h}, \sigma_{h}^{2}\right)$

$$
\begin{aligned}
& \propto N\left(\beta_{i, h} \mid \mu_{h}, \sigma_{h}^{2}\right) \prod_{t=1}^{T} \prod_{l=1}^{L_{t}} N\left(y_{t, i, l} \mid x_{t, i}+\sum_{s \neq h} \beta_{s} U_{l, s}+\beta_{h} U_{l, h}, R_{i}\right) \\
& \propto \quad N\left(\beta_{i, h} \mid \mu_{h}, \sigma_{h}^{2}\right) \prod_{t=1}^{T} \prod_{l=1}^{L_{t}} N\left(y_{t, i, l}-x_{t, i}+\sum_{s \neq h} \beta_{s} U_{l, s} \mid \beta_{h} U_{l, h}, R_{i}\right) \\
& \propto \quad N\left(\beta_{i, h} \mid \mu_{h}, \sigma_{h}^{2}\right) \prod_{t=1}^{T} \prod_{l=1}^{L_{t}} N\left[\frac{1}{U_{l, h}}\left(y_{t, i, l}-x_{t, i}+\sum_{s \neq h} \beta_{s} U_{l, s}\right) \mid \beta_{h}, \frac{R_{i}}{U_{l, h}^{2}}\right] \\
& \propto \quad N\left\{\omega\left[\frac{\mu_{h}}{\sigma_{h}^{2}}+\frac{1}{R_{i}} \sum_{t=1}^{T} \sum_{l=1}^{L_{t}} U_{l, h}\left(y_{t, i, l}-x_{t, i}-\sum_{s \neq h} \beta_{s} U_{l, s}\right)\right],\right. \\
& \left.\omega=\left(\frac{1}{\sigma_{h}^{2}}+\frac{1}{R_{i}} \sum_{t=1}^{T} \sum_{l=1}^{L_{t}} U_{l, h}^{2}\right)^{-1}\right\} .
\end{aligned}
$$


C.6. Sampling hyperparameters. The hyperparameters $\mu_{\lambda}, \sigma_{\lambda}^{2}, \mu_{a}, \sigma_{a}^{2}$ and $\mu_{h}, \sigma_{h}^{2}$ for hedonics $h=1, \ldots, H$ can be sampled as follows:

$\mu_{\lambda} \mid \mathbf{z}, \boldsymbol{\lambda}, \sigma_{\lambda}^{2}, \mu_{\lambda 0}, \sigma_{\lambda 0}^{2} \sim N\left[\omega\left(\frac{\mu_{\lambda 0}}{\sigma_{\lambda 0}^{2}}+\frac{1}{\sigma_{\lambda}^{2}} \sum_{k=1}^{K} \sum_{i: z_{i}=k} \lambda_{i k}\right), \omega=\left(\frac{1}{\sigma_{\lambda 0}^{2}}+\frac{p}{\sigma_{\lambda}^{2}}\right)^{-1}\right]$
$\sigma_{\lambda}^{2} \mid \mathbf{z}, \boldsymbol{\lambda}, \mu_{\lambda}, \alpha_{\lambda 0}, \beta_{\lambda 0} \sim \mathrm{IG}\left(\alpha_{\lambda 0}+\frac{p}{2}, \beta_{\lambda 0}+\frac{1}{2} \sum_{k=1}^{K} \sum_{i: z_{i}=k}\left(\lambda_{i k}-\mu_{\lambda}\right)^{2}\right)$

$$
\begin{aligned}
& \mu_{a} \mid\left\{a_{i}\right\}, \sigma_{a}^{2}, \mu_{a 0}, \sigma_{a 0}^{2} \sim N\left[\omega\left(\frac{\mu_{a 0}}{\sigma_{a 0}^{2}}+\frac{1}{\sigma_{a}^{2}} \sum_{i=1}^{p} a_{i}\right), \omega=\left(\frac{1}{\sigma_{a 0}^{2}}+\frac{p}{\sigma_{a}^{2}}\right)^{-1}\right] \\
& \sigma_{a}^{2} \mid\left\{a_{i}\right\}, \mu_{a}, \alpha_{a 0}, \beta_{a 0} \sim I G\left[\alpha_{a 0}+\frac{p}{2}, \beta_{a 0}+\frac{1}{2} \sum_{i=1}^{p}\left(a_{i}-\mu_{a}\right)^{2}\right]
\end{aligned}
$$

$$
\begin{aligned}
& \mu_{h} \mid \beta_{1: p, h}, \sigma_{h}^{2}, \mu_{h 0}, \sigma_{h 0}^{2} \sim N\left[\omega\left(\frac{\mu_{h 0}}{\sigma_{h 0}^{2}}+\frac{1}{\sigma_{h}^{2}} \sum_{i=1}^{p} \beta_{i, h}\right), \omega=\left(\frac{1}{\sigma_{r 0}^{2}}+\frac{p}{\sigma_{r}^{2}}\right)^{-1}\right] \\
& \sigma_{h}^{2} \mid \beta_{1: p, h}, \mu_{h}, \alpha_{h 0}, \beta_{h 0} \sim \mathrm{IG}\left[\alpha_{h 0}+\frac{p}{2}, \beta_{h 0}+\frac{1}{2} \sum_{i=1}^{p}\left(\beta_{i, h}-\mu_{h}\right)^{2}\right] .
\end{aligned}
$$

C.7. Sampling the DP hyperparameter $\boldsymbol{\alpha}$. Following Escobar and West (1994), we assume a gamma distribution prior for the concentration parameter $\alpha \sim \operatorname{Gamma}\left(\alpha_{\alpha}, \beta_{\alpha}\right)$. We sample an auxiliary variable $\kappa$ to help us sample $\alpha$ :

1. Sample $\kappa \sim \operatorname{Beta}(\alpha+1, n)$, where $n$ is the total number of tracts.

2. Sample $\alpha$ from the a mixture of two gamma distributions as follows:

$$
\begin{aligned}
\alpha \mid \kappa, K \sim & \pi \operatorname{Gamma}\left(\alpha_{\alpha}+K, \beta_{\alpha}-\log (\kappa)\right) \\
& +(1-\pi) \operatorname{Gamma}\left(\alpha_{\alpha}+K-1, \beta_{\alpha}-\log (\kappa)\right)
\end{aligned}
$$

where $K$ is the number of unique clusters, and the mixture weight $\pi$ is defined by $\pi /(1-\pi)=\left(\alpha_{\alpha}+K-1\right) /\left(p\left[\beta_{\alpha}-\log (\kappa)\right]\right)$. 


\section{APPENDIX D: PARALLEL DPMM SAMPLER}

Sampling the cluster membership $z_{i}$ in parallel includes the following two steps:

1. Local step on each machine in parallel:

Conditioned on the processor assignments $\gamma$, we sample the cluster assignments $\left\{z_{i}: \gamma_{i}=j\right\}$ as in a conventional Dirichlet process mixture model (Section 4.1 of the main paper) with concentration parameter $\alpha / P$, for data points assigned to a machine $j$. Since the DPMMs are independent given the processor allocations, we can sample $\left\{z_{i}: \gamma_{i}=\right.$ $j\}$ in parallel across machines.

2. Global step over machines:

Each cluster is associated with a single processor. One processor can have multiple clusters. We jointly resample the processor allocations of all data points within a given cluster. We use a Metropolis-Hastings step with a proposal distribution that independently assigns cluster $k$ to processor $j$ with probability $1 / P$. This means our accept/reject ratio depends only on the ratio of the likelihoods of the current processor assignments $\left\{\gamma_{i}\right\}$ and the proposal $\left\{\gamma_{i}^{*}\right\}$.

The likelihood ratio is given by:

$$
\begin{aligned}
\frac{p\left(\left\{\gamma_{i}^{*}\right\}\right)}{p\left(\left\{\gamma_{i}\right\}\right)} & =\frac{p\left(\left\{z_{i}\right\} \mid \gamma_{i}^{*}\right) p\left(\left\{\gamma_{i}^{*}\right\} \mid \alpha, P\right)}{p\left(\left\{z_{i}\right\} \mid \gamma_{i}\right) p\left(\left\{\gamma_{i}\right\} \mid \alpha, P\right)} \\
& =\prod_{j=1}^{P} \prod_{i=1}^{\max \left(N_{j}, N_{j}^{*}\right)} \frac{a_{i j} !}{a_{i j}^{*} !}
\end{aligned}
$$

where $N_{j}$ is the number of data points on machine $j$, and $a_{i j}$ is the number of clusters of size $i$ on machine $j$. The derivation is shown in the supplementary material of Williamson et al. (2013).

\section{APPENDIX E: HYPERPRIOR SETTINGS}

Our hyperpriors are defined by a set of parameters:

- $\left(\alpha_{\epsilon 0}, \beta_{\epsilon 0}\right)$ for the innovation idiosyncratic noise variance parameter $\sigma_{0}^{2}$

- $\left(\alpha_{R 0}, \beta_{R 0}\right)$ for the observation noise variance parameter $R_{i}$

- $\left(\mu_{\lambda 0}, \sigma_{\lambda 0}^{2}, \alpha_{\lambda 0}, \beta_{\lambda 0}\right)$ for the factor loading hyperparameters $\left(\mu_{\lambda}, \sigma_{\lambda}^{2}\right)$

- $\left(\mu_{a 0}, \sigma_{a 0}^{2}, \alpha_{a 0}, \beta_{a 0}\right)$ for the autoregressive hyperparameters $\left(\mu_{a}, \sigma_{a}^{2}\right)$

- $\left(\mu_{h 0}, \sigma_{h 0}^{2}, \alpha_{h 0}, \beta_{h 0}\right)$ for the hedonic regression hyperparameters $\left(\mu_{h}, \sigma_{g}^{2}\right)$.

E.1. Hyperprior for $\boldsymbol{\sigma}_{\mathbf{0}}^{\mathbf{2}}$. We set the hyper priors for $\sigma_{0}^{2} \sim \operatorname{IG}\left(\alpha_{\epsilon 0}, \beta_{\epsilon 0}\right)$ with hyperparameters $\alpha_{\epsilon 0}=0.5, \beta_{\epsilon 0}=1$. When examining the housing data, 
for numerical stability we multiply the observations $\left[\log \left(\right.\right.$ Price $\left.\left._{t, i, l}\right)-\log \left(g_{t}\right)\right]$ by a factor of 200 . As a result, $99 \%$ of outcome values are covered by the interval $[-1.10,1.61]$. The chosen hyper prior has a long and flat tail distribution over the range of variance.

E.2. Hyperprior for $\boldsymbol{R}_{\boldsymbol{i}}$. We set the hyper priors for $R_{i} \sim \operatorname{IG}\left(\alpha_{R 0}, \beta_{R 0}\right)$ with hyperparameters $\alpha_{R 0}=3, \beta_{R 0}=1$. The chosen hyper prior has a long and flat tail distribution over the range of variance.

E.3. Hyperpriors for the factor loading, autoregressive, and hedonic regression hyperparameters. We set the prior parameters as $\mu_{\lambda 0}=3, \sigma_{\lambda 0}^{2}=2^{2}, \alpha_{\lambda 0}=0.1, \beta_{\lambda 0}=0.1, \mu_{a 0}=0.95, \sigma_{a 0}^{2}=0.1^{2}, \alpha_{a 0}=$ $0.2, \beta_{a 0}=\frac{1}{200^{2}}, \mu_{h 0}=0, \sigma_{h 0}^{2}=200^{2}, \alpha_{h 0}=0.1, \beta_{h 0}=0.1$. The fact that some of our prior parameters are set as a factor of 200 is motivated by the fact that, for our housing data, the observations $\left[\log \left(\right.\right.$ Price $\left.\left._{t, i, l}\right)-\log \left(g_{t}\right)\right]$ are multiplied by a factor of 200. Again, this is done for numerical stability.

\section{APPENDIX F: FURTHER DETAILS ON SIMULATED DATA EXPERIMENTS}

F.1. Simulation settings. We simulated 20 data streams corresponding to sales in 20 census tracts from January 1997 to September 2013, a period of 213 months. The 20 tracts are pre-assigned to four clusters of size 4, 4, 4 and 8 census tracts, respectively. These assignments determine the set of cluster indicators $z_{i}$.

First, we generated latent price processes, $x_{1: T, i}$, for each tract according to Eqs. (3.3) and (3.5) (see Figure 5). The autoregressive coefficients $a_{i}$ for the 20 latent processes in Eq. (3.3) were simulated i.i.d. from $N\left(0.99,0.005^{2}\right)$. For the value of $k$ such that $z_{i}=k$, the innovations $\epsilon_{t, i}$ were constructed from factor loading $\lambda_{i k}$, cluster-specific latent factor $\eta_{t, k}^{*}$ and idiosyncratic noise $\tilde{\epsilon}_{t, i}$. We simulated these three components from the following distributions for $k=1, \ldots, 4, \quad t=1, \ldots, 213, \quad i=1, \ldots, 20$ :

$$
\begin{aligned}
\lambda_{i k} & \sim \mathcal{N}\left(0.015,0.002^{2}\right), \\
\eta_{t, k}^{*} & \sim \mathcal{N}(0,1), \\
\tilde{\epsilon}_{t, i} & \sim \mathcal{N}\left(0, \sigma_{0}^{2}=0.01^{2}\right) .
\end{aligned}
$$

The parameters in the simulation distribution were based on exploratory analysis on the transaction data in City of Seattle.

Second, we generated the observed sales prices, $y_{t, i, l}$, according to Eq. (3.4). The sales dates and house hedonics are taken from 20 randomly sampled tracts in the City of Seattle, so as to match the real-data frequency of 
observations and house characteristics. Among the 20 sampled census tracts, the transaction frequency ranges from 2 sales to 2157 sales over 17 years, with a median monthly sales of 3.7. Table 1 shows the distribution of transaction frequencies in the simulated data. Note that, by construction, these numbers match very closely with the distribution of transaction frequency in the overall Seattle data in Table 1.

TABLE 1

Number of census tracts in simulated data that have less than single digit transactions per month on average.

\begin{tabular}{lrrrrr}
\hline Average monthly sales & $<1$ & $<3$ & $<5$ & $<7$ & $<9$ \\
\hline Number of tracts & 3 & 8 & 16 & 18 & 19 \\
Proportion of tracts & 0.15 & 0.40 & 0.80 & 0.90 & 0.95 \\
\hline
\end{tabular}

The hedonic effect $\beta_{i, h}$ in Eq. (3.5) for each tract and covariate is simulated i.i.d from $\mathcal{N}\left(0.1,0.01^{2}\right)$ except for the intercept. The intercept is simulated from $\mathcal{N}\left(0.1,0.02^{2}\right)$. The choice of parameters are based on exploratory analysis on the empirical data of Seattle City.

F.2. Extended simulation results. In this section, we provide a performance analysis of the remaining clusters not examined in Section 5.2 of the main paper. Figures 1,2 and 3 directly parallel Figure 8 of the main paper and show our performance in estimating the simulated intrinsic price dynamics compared to an independent Kalman-filter-based analysis of the tracts.

We also designed another simulation setting of only one big cluster. The true latent trends for the 20 tracts are shown in Figure 4 (left). We are able to recover the true clustering structure - one big cluster - as indicated in Figure 4 (right), which shows the posterior distribution of the total number of clusters. We see the posterior mass concentrated at having one cluster. Being a Bayesian nonparametric method, there will always be some mass on having more clusters.

\section{APPENDIX G: EXTENDED SEATTLE CITY RESULTS}

In this section, we present further details and figures from our Seattle City data analysis to augment those presented in the main paper.

G.1. Global trend estimation. We first provide details on our global trend estimation procedure used in Section 6. For the precomputed global trend of that section, we use an estimate formed as follows. We first consider a non-tract-specific regression akin to Eq. (3.2) in order to remove the hedonic effects: $\tilde{y}_{t, i, l}=\alpha_{0}+\sum_{t} \alpha_{t} I(t)+\sum_{h=1}^{H} \beta_{h} U_{l, h}+v_{t, i, l}$, where $\alpha_{t}$ captures 

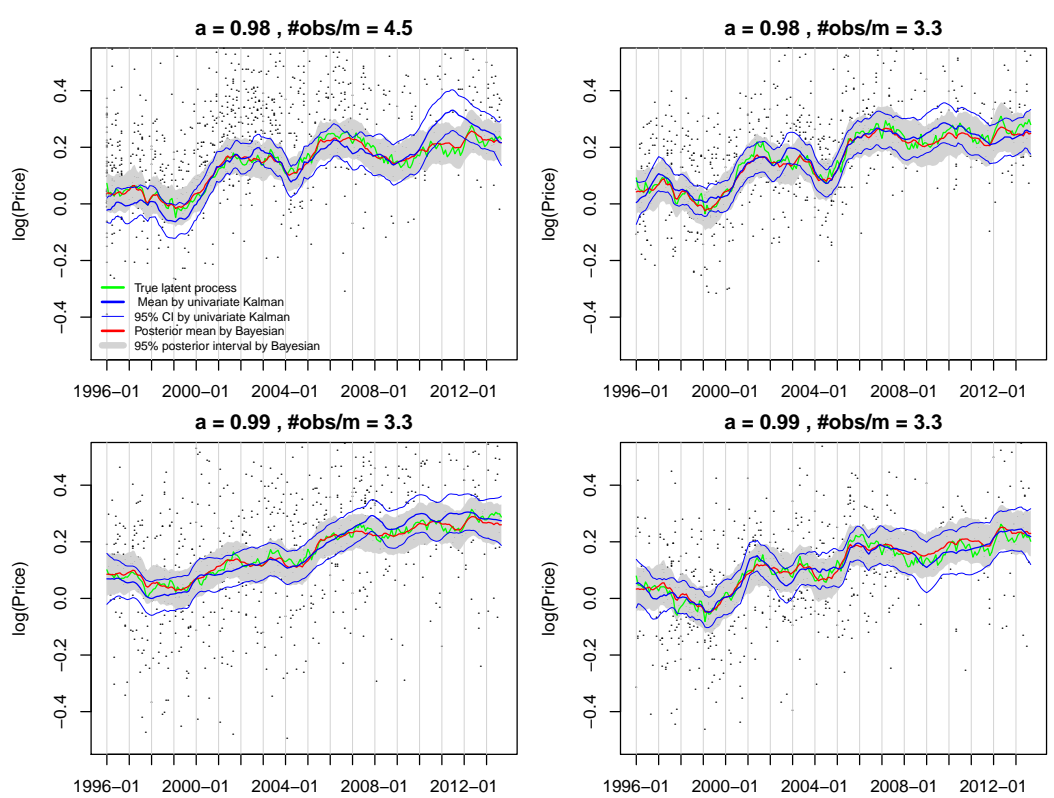

FIG 1. Performance of estimating the latent process for Cluster 2. Comparisons are as in Figure 8 of the main paper.

the monthly effect and $\beta_{h}$ the hedonic effects on the global trend. The noise $v_{t, i, l}$ is independent across time, tracts, and sales. Note that in aggregate, we have roughly 640 observations per month on average. After removing the hedonic effects, we then apply the seasonal decomposition approach of Cleveland et al. (1990) to decompose the estimated global trend into a trend component, seasonal component, and noise; we discard the noise term. We then remove our estimated $g_{t}$ from the observations to form $y_{t, i, l}=\tilde{y}_{t, i, l}-g_{t}$ used in Eq. (3.4).

G.2. Cluster trends. For the MAP MCMC sample of our analysis in Section 6, Figure 5 displays the average of the intrinsic price processes within a cluster, for each of the 16 inferred clusters. This plot parallels that of Figure 11 of the main paper, but here in the raw price space instead of log space and with the global trend added without the seasonality component (for clarity). We additionally hold on the estimated global trend without seasonality for comparison. 

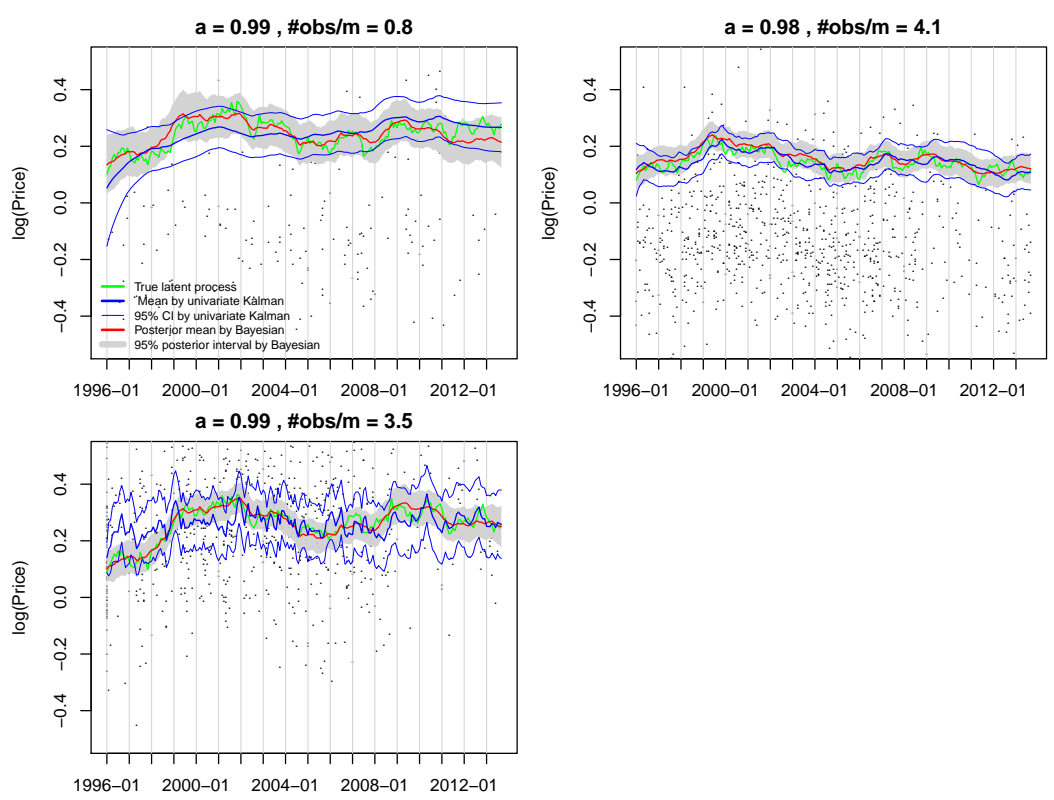

FIG 2. Performance of estimating the latent process for Cluster 3. Comparisons are as in Figure 8 of the main paper.

G.3. Sales volume and variance over time. Figure 6 shows the sales volume and its variance over time, as discussed in Section 6.1, together with Figure 13, of the main paper. The market boom, roughly 2006-2007, and subsequent stabilization, roughly 2010-2011, were manifested in the different housing sectors in disparate ways. The index formed from the model based on DP clustering is able to capture the dynamics of the change in value for different housing sectors during these two periods.

G.4. Zip code level comparison of models. To further clarify the visualization of results in Figures 13 and 14 of the main paper, in Figures 7 and 8 we provide a 3 -panel representation with each method's performance displayed in its own panel.

G.5. Neighborhood price index. In contrast to existing index models whose performance typically degrades when moving to finer resolutions, in this section we show that we can achieve even better performance at granularities below that of census tracts. Ren (2015) studied the problem of defining neighborhood structure for residential houses. Based on the idea 
SUPPLEMENTARY MATERIAL FOR MODELING A HYPERLOCAL HOUSING PRICE INDER

TABLE 2

Predictive performance comparison of the Bayesian nonparametric housing index approach applied to census tracts and discovered neighborhoods. Note that the neighborhoods are at a finer granularity than the census tracts.

\begin{tabular}{lrr}
\hline & Bayesian & Bayesian \\
& Census Tract Index & Neighborhood Index \\
\hline RMSE & 122,026 & 120,198 \\
Mean APE & 0.1633 & 0.1565 \\
Median APE & 0.1231 & 0.1165 \\
90th APE & 0.3422 & 0.3208 \\
P10 & 0.4183 & 0.4392 \\
\hline
\end{tabular}

that the definition of a neighborhood should incorporate housing and road characteristics, Ren (2015) utilized road network data to guide the partitioning of space into neighborhoods. In particular, the method encodes spatial relationships through a graphical network where each node denotes a street segment along with housing and road features, and each edge denotes connectivity between two street segments. A cost minimization algorithm was designed to optimize for within-neighborhood housing heterogeneity, following natural boundaries defined by road networks while regularizing for neighborhood sizes. The discovered neighborhood structure in Seattle City depicted in Figure 9 shows results consistent with commonly recognized neighborhoods.

In our analysis, Seattle has 140 census tracts whereas the cost minimization algorithm of Ren (2015) yields 259 neighborhoods (under the setting of the desired neighborhood size containing between 400 to 600 houses). Hence, these estimated neighborhoods are at a finer granularity than that of census tracts.

We applied the proposed Bayesian dynamical model of Section 3 to house sales time series associated with the discovered neighborhoods. Even at this hyperlocal level, the Bayesian nonparametric model is able to provide a robust housing price index for neighborhoods. This is demonstrated by the predictive performance summarized in Table 2 . We see that by capturing hyperlocal dynamics that might otherwise be blurred even at the census tract level, the neighborhood index outperforms the census tract index in all metrics. This performance gain relies on the fact that our method is able to cope with the even greater degree of data scarcity at this hyperlocal level. In contrast, recall that the performance of the Case-Shiller index, for example, degraded when moving from city to zip code level, and could not even scale to census tracts let alone the fine scale of these discovered neighborhoods. These results demonstrate the robustness of our proposed approach. 


\section{APPENDIX H: MODEL SELECTION, PRIOR AND POSTERIOR FOR THE JOINT MODEL WITH GLOBAL TREND}

H.1. Exploratory Analysis for the Global Trend. We examine posterior samples of the residuals $r_{t, i, l}$ computed as in Eq. (7.2) based on our MCMC samples of $x_{t, i, l}$ and $\beta_{i, h}$ using the model in Section 3 . Figure 10 shows the fit of the seasonal global trend model in Eq. (7.1) with different number of knots for the natural cubic spline (NCS) model of Eq. (7.1). We clearly see that the global trend exhibits monthly effects within each year. Figure 11 shows the estimated smoothed global trend after removing the monthly effects, in order to visualize the fit of the NCS basis functions with different number of knots alone. With too few knots, for example three, the curve is more constrained and the fit is unsatisfactory. The model selection procedure suggests using 9 interior knots, according to the Bayesian Information Criterion (BIC), as shown in Figure 12. The 9 interior knots generate 10 spline basis functions and one intercept basis. Therefore, in our experiments we use $N_{B}=11$.

H.2. Prior Specification for Global Trend. In this section, we focus on the non-stationary smooth trend over the long term horizon, as modeled by natural cubic splines (NCS), assuming the monthly effects are preestimated and removed. The priors for the coefficients of NCS are specified as

$$
w_{j} \sim \mathcal{N}\left(0, \sigma_{w_{j}}^{2}\right), \quad j=1, \cdots, N_{B}
$$

The hyperpriors are specified as

$$
\sigma_{w_{j}}^{2} \sim \operatorname{IG}\left(\alpha_{w 0}, \beta_{w 0}\right), \quad j=1, \cdots, N_{B}
$$

H.3. MCMC Posterior Computation with Global Trend. Our posterior computation is implemented via a Gibbs sampling scheme building directly on the sampler derived in Section 4. Define $\mathbf{g}=\left\{g_{t}\right\}, \mathbf{w}=\left\{w_{j}\right\}$ and $\mathbf{s}=\left\{s_{j}\right\}$. The global trend $\mathbf{g}$ is a function of the parameters $\mathbf{w}$ and $\mathbf{s}$. Let $\boldsymbol{\psi}=\left\{\mathbf{a}=\left\{a_{i}\right\}, \boldsymbol{\lambda}=\left\{\lambda_{i k}\right\}, \mathbf{R}=\left\{R_{i}\right\}, \boldsymbol{\beta}=\left\{\beta_{i, h}\right\}, \sigma_{0}^{2}\right\}$ and $\boldsymbol{\psi}^{(k)}$ be the associated subset of parameters corresponding to the $k$-th cluster based on assignments $\mathbf{z}=\left\{z_{i}\right\}$.

Based on the local latent dynamics $\mathbf{x}$ and house hedonic effects $\boldsymbol{\beta}$, we can form the residuals $\mathbf{r}=\left\{r_{t, i, l}\right\}$, according to $r_{t, i, l}=\tilde{y}_{t, i, l}-x_{t, i}-\sum_{h=1}^{H} \beta_{i, h} U_{l, h}$. Given these pseudo-observations $\mathbf{r}$, we then sample the conditional posterior of the global trend $\mathbf{g}$. Our Gibbs sampler is outlined as follows:

imsart-aoas ver. 2014/02/20 file: Appendix.tex date: March 27, 2017 
1. Sample $z_{i}=k \mid \mathbf{z}_{-i}, \alpha, \tilde{\mathbf{y}}, \boldsymbol{\psi}, \mathbf{g}$.

2. Impute $\mathbf{x}$ and $\boldsymbol{\eta}^{*}$ as auxiliary variables. Specifically, block sample $\mathbf{x}, \boldsymbol{\eta}^{*}$ as $\mathbf{x}^{(k)} \mid \mathbf{z}, \tilde{\mathbf{y}}^{(k)}, \boldsymbol{\psi}^{(k)}, \mathbf{g}$ and $\boldsymbol{\eta}^{*} \mid \mathbf{z}, \mathbf{x}, \boldsymbol{\psi}$.

3. Sample $\boldsymbol{\psi}^{(k)} \mid \mathbf{z}, \tilde{\mathbf{y}}^{(k)}, \mathbf{x}^{(k)}, \boldsymbol{\eta}^{*(k)}, \mathbf{g}$.

4. Obtain a sample of $\mathbf{g}$ by sampling $\mathbf{w} \mid \tilde{\mathbf{y}}, \mathbf{x}, \boldsymbol{\psi}, \mathbf{s}$.

5. Discard $\mathbf{x}$ and $\boldsymbol{\eta}^{*}$ to sample hyperparameters conditional on $\boldsymbol{\psi}, \mathbf{z}$.

Steps 1, 2, 3, and 5 follow exactly as in Section 4, first forming $y_{t, i, l}=$ $\tilde{y}_{t, i, l}-g_{t}$ if $y_{t, i, l}$ is needed. Step 4 is detailed in Supplement H.4.

H.4. Sampling the coefficients of the global trend NCS. By the model specification of the global trend in Eq. (7.3) and Eq. (7.1),

$$
r_{t, i, l}=\mathbf{w}_{-j}^{T} \mathbf{B}_{-j}(t)+w_{j} B_{j}(t)+\mathbf{s}^{T} \mathbf{m}(t)+v_{t, i, l}, \quad v_{t, i, l} \sim \mathcal{N}\left(0, R_{i}\right) .
$$

Therefore,

$$
r_{t, i, l} \sim \mathcal{N}\left(\mathbf{w}_{-j}^{T} \mathbf{B}_{-j}(t)+w_{j} B_{j}(t)+\mathbf{s}^{T} \mathbf{m}(t), R_{i}\right) .
$$

Rearranging the terms,

$$
\frac{r_{t, i, l}-\mathbf{w}_{-j}^{T} \mathbf{B}_{-j}(t)-\mathbf{s}^{T} \mathbf{m}(t)}{B_{j}(t)} \sim \mathcal{N}\left(w_{j}, \frac{R_{i}}{B_{j}^{2}(t)}\right), \quad \text { i.i.d. across } l .
$$

By conjugacy, the full conditional distribution for the coefficients of the global trend NCS is

$$
\begin{gathered}
p\left(w_{j} \mid \mathbf{r}, \mathbf{s}, \mathbf{R}, \sigma_{w_{j}}^{2}\right) \propto \mathcal{N}\left(w_{j} \mid 0, \sigma_{w_{j}}^{2}\right) \prod_{t, i, l} \mathcal{N}\left(\frac{r_{t, i, l}-\mathbf{w}_{-j}^{T} \mathbf{B}_{-j}(t)-\mathbf{s}^{T} \mathbf{m}(t)}{B_{j}(t)} \mid w_{j}, \frac{R_{i}}{B_{j}^{2}(t)}\right) \\
\quad \propto \mathcal{N}\left\{\begin{array}{c}
\omega\left[\sum_{i=1}^{p} \frac{\sum_{t, l} B_{j}\left(t_{t, i, l}\right) \cdot\left(r_{t, i, l}-\mathbf{w}_{-j}^{T} \mathbf{B}_{-j}(t)-\mathbf{s}^{T} \mathbf{m}(t)\right)}{R_{i}}\right], \\
\omega=\left[\frac{1}{\sigma_{w_{j}}^{2}}+\sum_{i=1}^{p} \frac{\sum_{t, l} B_{j}^{2}\left(t_{t, i, l}\right)}{R_{i}}\right]^{-1}
\end{array}\right\} .
\end{gathered}
$$

\section{REFERENCES}

Cleveland, R. B., W. S. Cleveland, J. E. McRae, and I. Terpenning (1990). STL: A seasonal-trend decomposition procedure based on loess (with discussion). Journal of Official Statistics 6, 3-73.

Escobar, M. D. and M. West (1994). Bayesian density estimation and inference using mixtures. Journal of the American Statistical Association 90, 577-588. 
Ren, Y. (2015). Bayesian Modeling of a High Resolution Housing Price Index. Ph.D. thesis, University of Washington.

Williamson, S., A. Dubey, and E. Xing (2013). Parallel Markov chain Monte Carlo for nonparametric mixture models. In International Conference on Machine Learning, pp. 98-106.

You Ren, Emily B. Fox, and Andrew Bruce Department of Statistics

UNIVERSITY OF WASHINGTON

Box 354322

Seattle, WA 98195

USA

E-MAIL: shirleyr@uw.edu ebfox@stat.washington.edu

andrewb0@uw.edu 

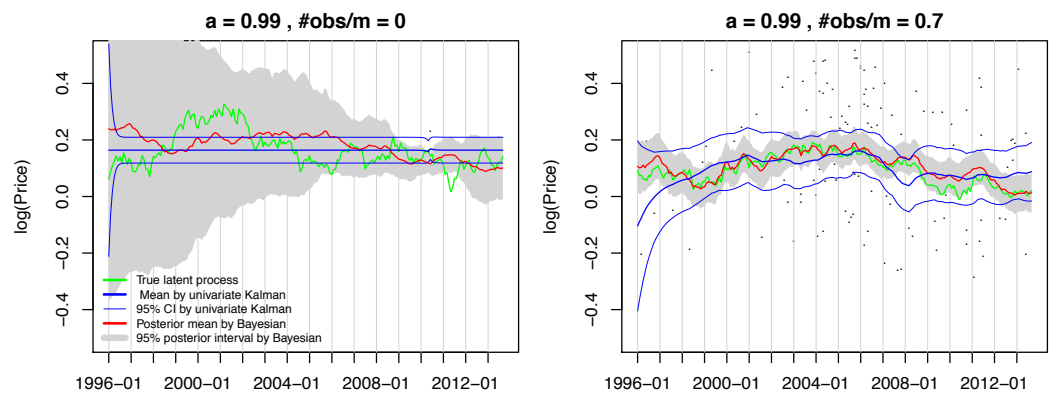

1996-01 2000-01 2004-01 2008-01 2012-01 $\mathrm{a}=0.99, \# \mathrm{obs} / \mathrm{m}=6.2$
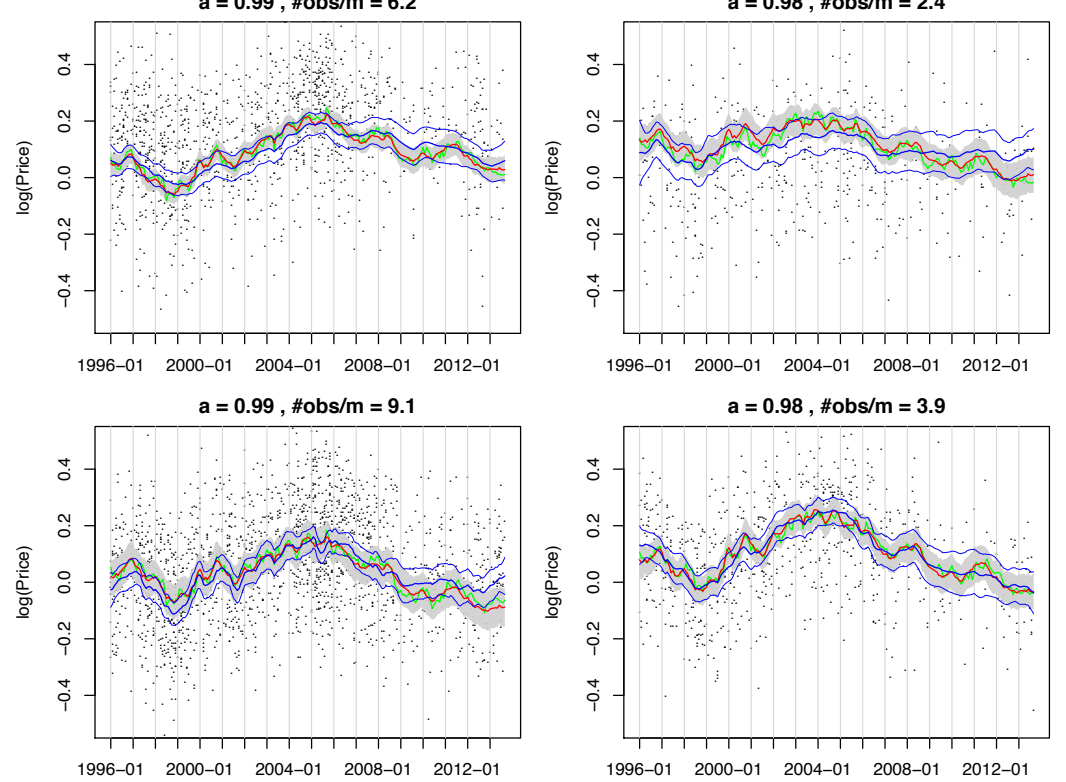

$\mathrm{a}=0.99, \# \mathrm{obs} / \mathrm{m}=1.1$

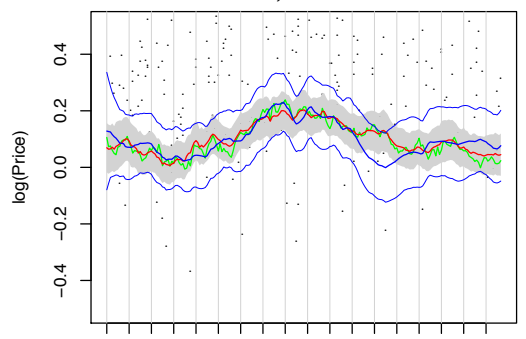

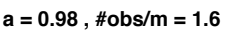
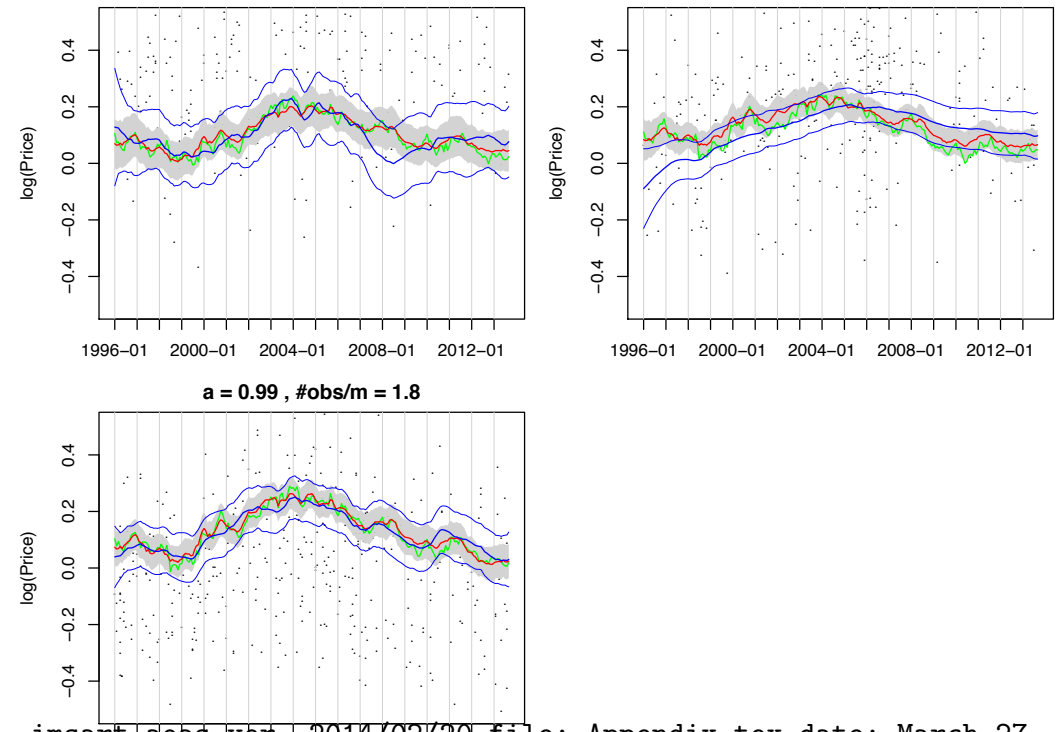

imsarttaoas vert 2014/021/20 file: Appendix.tex date: March 27, 2017

FIG 3. Performance of estimating the latent process for Cluster 4. Comparisons are as in Figure 8 of the main paper. 

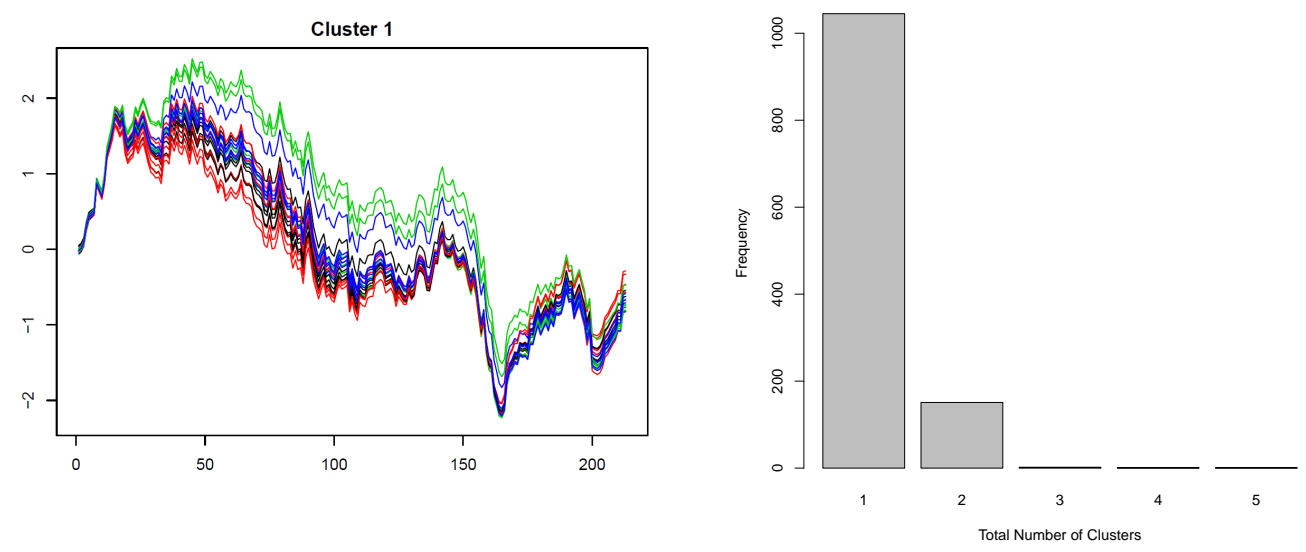

FIG 4. Left: Simulated latent trends for 20 tracts from one big cluster. Right: Posterior samples of total number of clusters. 

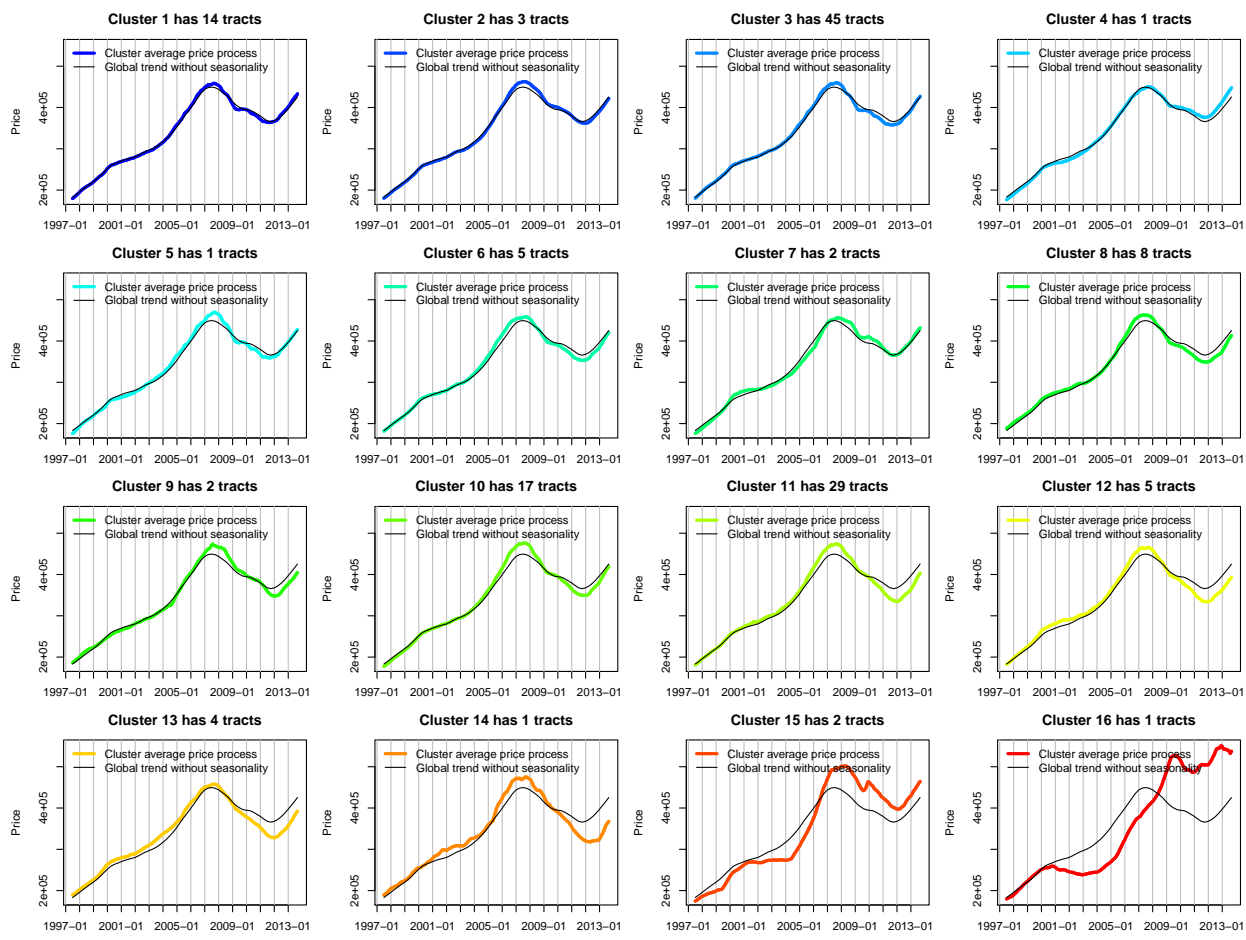

FIG 5. Under the MAP sample, we first compute the cluster-average intrinsic price dynamics by averaging $x_{1: T, i}$ over all $i$ with $z_{i}=k$ for $k=1, \ldots, 16$ (all of the estimated clusters). We then add this cluster-average price to the global trend without seasonality (various colors) and hold on the seasonally adjusted global trend (black) for comparison. 

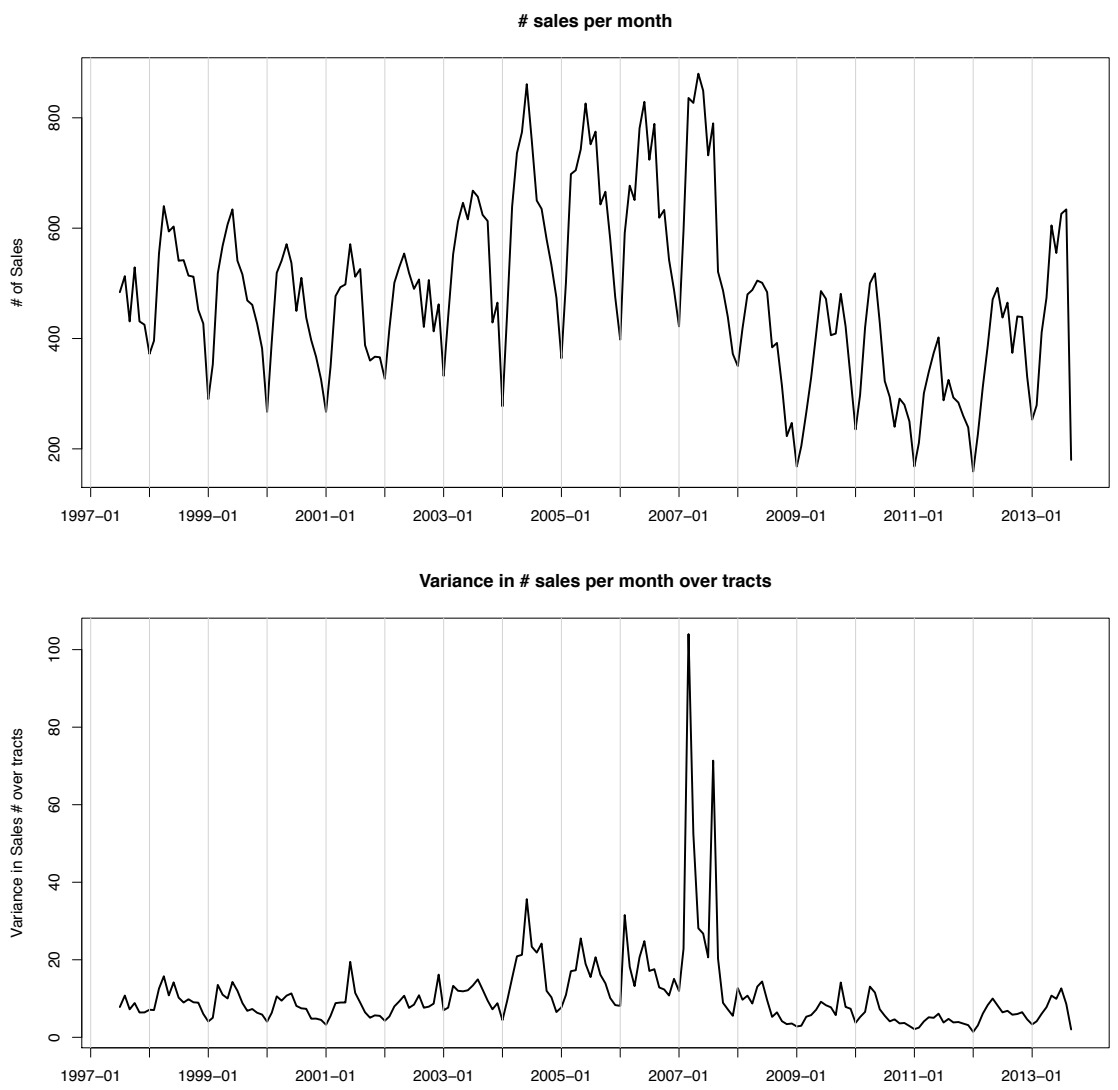

FIG 6. Sales volume (top) and variance (bottom) versus time. 


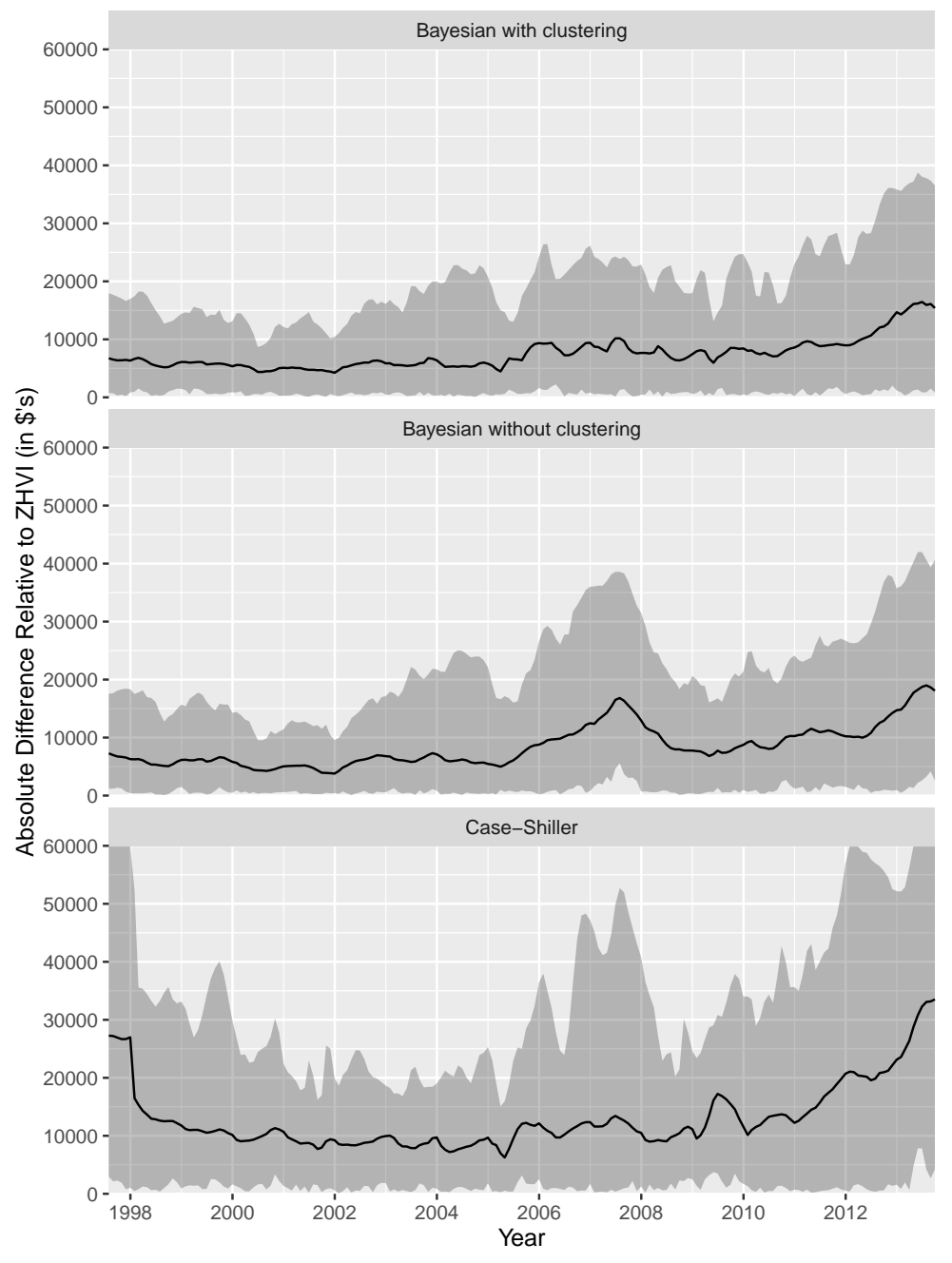

FIG 7. As in Figure 13, but here with results broken down into 3 separate panels, one for each method. 


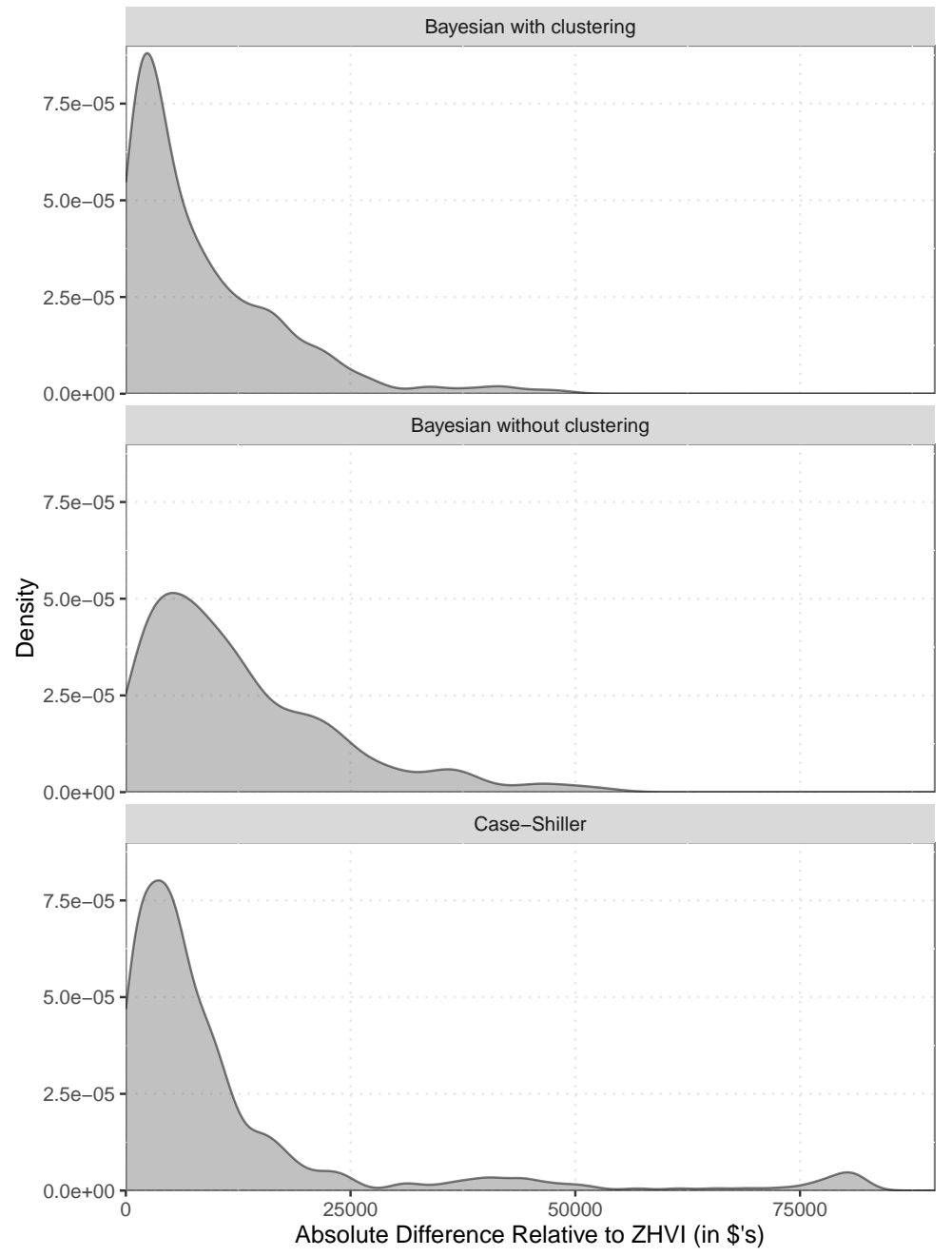

FIG 8. As in Figure 14, but here with results broken down into 3 separate panels, one for each method.

imsart-aoas ver. 2014/02/20 file: Appendix.tex date: March 27, 2017 


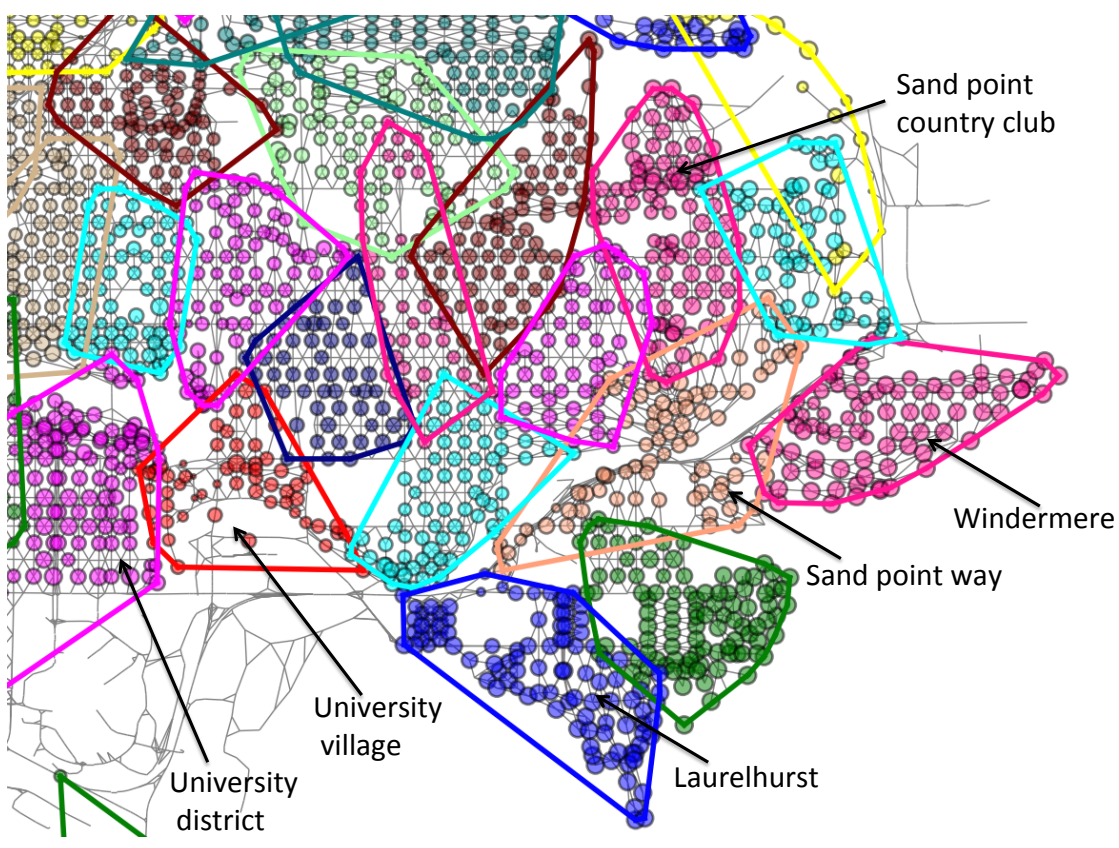

FIG 9. Discovered neighborhoods of Ren (2015), which are shaped by house features, road types and connectivity. These discovered neighborhoods are consistent with real Seattle neighborhoods, as labeled in this figure. 


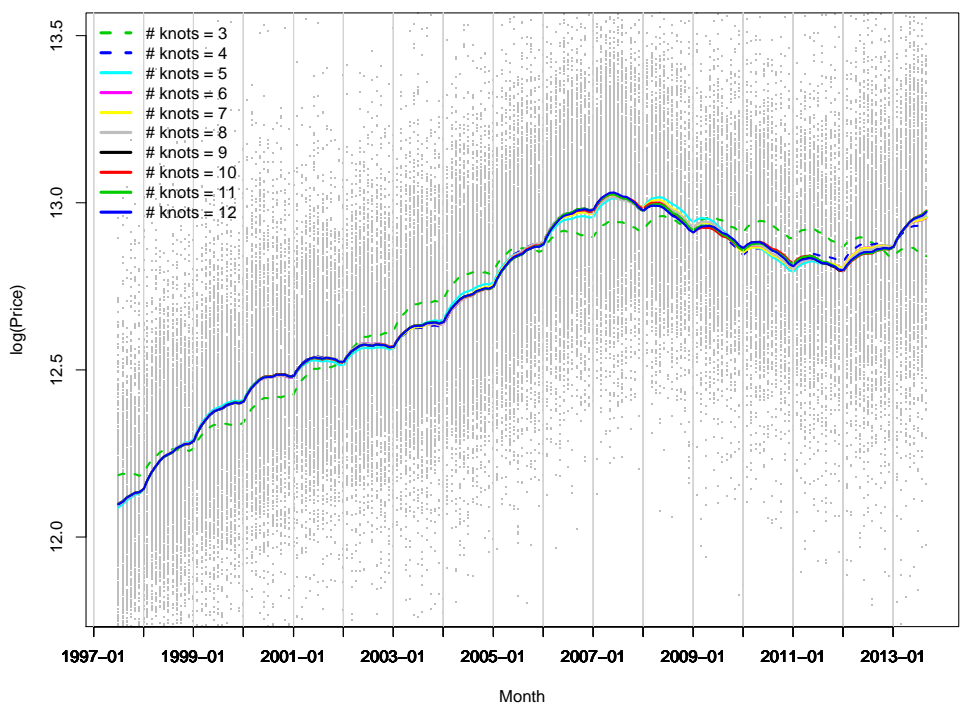

FIG 10. The fit of the seasonal global trend model in Eq. (".1) with different number of knots for the NCS. We clearly see monthly effects exhibited within each year.

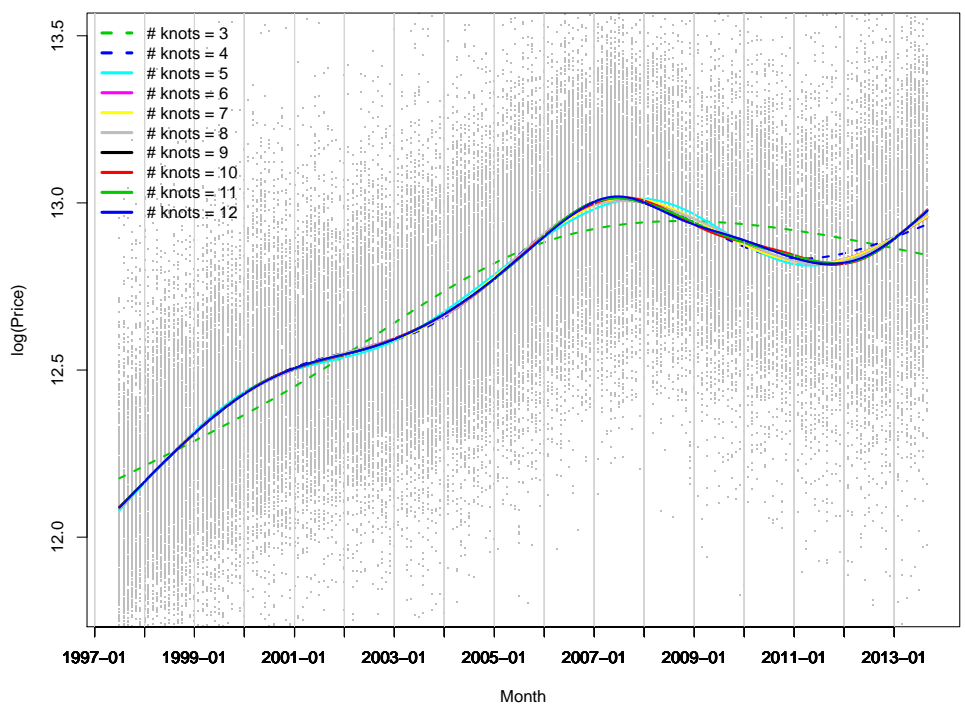

FIG 11. The estimated smooth global trend after removing the monthly effects, for visualization of the NCS basis fit alone as a function of the number of knots.

imsart-aoas ver. 2014/02/20 file: Appendix.tex date: March 27, 2017 


\section{SUPPLEMENTARY MATERIAL FOR MODELING A HYPERLOCAL HOUSING PRICE INDE2}

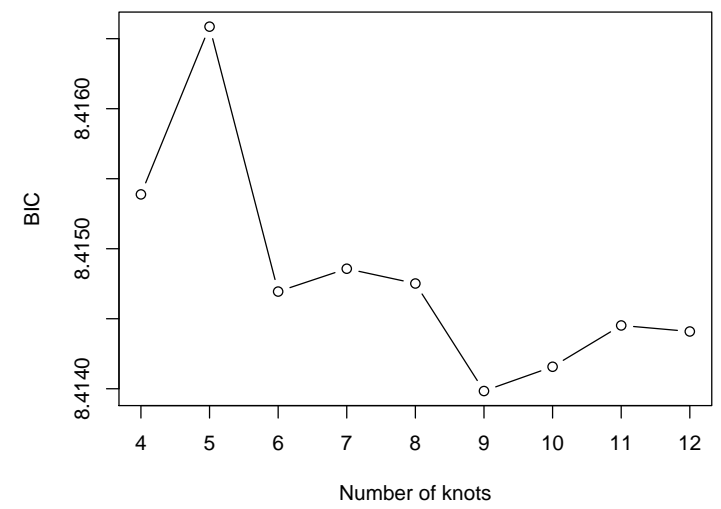

FIG 12. Model selection of the number of knots by BIC for the global trend NCS model with monthly effects. 